九州大学学術情報リポジトリ

Kyushu University Institutional Repository

\title{
Magnetic separation of cells from developing embryoid bodies using magnetite cationic liposomes
}

Horie, Masanobu

Department of Chemical Engineering, Faculty of Engineering, Kyushu University

Ito, Akira

Department of Chemical Engineering, Faculty of Engineering, Kyushu University

Maki, Takeshi

Department of Chemical Engineering, Faculty of Engineering, Kyushu University

Kawabe, Yoshinori

Department of Chemical Engineering, Faculty of Engineering, Kyushu University

他

http://hdl. handle. net/2324/25611

出版情報: Journal of Bioscience and Bioengineering. 112 (2), pp.184-187，2011-08-02. Elsevier バージョン:

権利関係: (C) 2011, The Society for Biotechnology, Japan. 


\title{
Magnetic separation of cells from developing embryoid bodies using magnetite cationic liposomes
}

\author{
Masanobu Horie, Akira Ito, Takeshi Maki, Yoshinori Kawabe, and Masamichi Kamihira* \\ Department of Chemical Engineering, Faculty of Engineering, Kyushu University, 744 Motooka, \\ Nishi-ku, Fukuoka 819-0395, Japan
}

Key words: embryonic stem cells, embryoid body, germ cells, magnetic separation, magnetic force

\footnotetext{
*Corresponding author: Masamichi Kamihira

E-mail: kamihira@chem-eng.kyushu-u.ac.jp
}

Tel: +81-92-802-2743: Fax: +81-92-802-2793 
Abstract

Embryoid bodies resemble post-implantation egg-cylinder stage embryos and are used to differentiate embryonic stem cells in vitro. In this study, we enriched mouse vasa homolog-positive germ cells from embryoid bodies after 8 days of differentiation using a magnetic separation method with magnetite cationic liposomes. 
Mouse embryonic stem (ES) cells are pluripotent cells of an embryonic origin. Once ES cells are removed from anti-differentiation factors, such as leukemia inhibitory factor (LIF) or feeder cells such as mouse embryonic fibroblasts (MEFs), they spontaneously differentiate into endoderm, mesoderm and ectoderm germ layers (1). The most robust method for the differentiation of cell types is the formation of embryoid bodies (EBs) (2), in which ES cells spontaneously form three-dimensional multicellular aggregates of differentiated and undifferentiated cells similar to those in embryos at the egg-cylinder stage. Target cells can be isolated from EBs for characterization of specific cell types. Magnetic cell sorting is a simple method for preparative cell isolation, and widely used in research and clinical applications (3). Magnetic particles most frequently used for magnetic cell sorting are ferrites including magnetite $\mathrm{Fe}_{3} \mathrm{O}_{4}$. Magnetite cationic liposomes (MCLs) contain $10 \mathrm{~nm}$ magnetite nanoparticles and use the electrostatic interactions between MCLs and the cell membrane for magnetic labeling of cells $(4,5)$. Various cell types can be labeled with MCLs because the cell surface is negatively charged. Based on MCL-labeled cell manipulation using a magnet, we reported the magnetic separation of target cells in co-culture systems using MCLs (6). In this method, mouse ES cells were co-cultured with STO feeder cells labeled with MCLs, which were magnetically removed from the target ES cells. Cell proliferation was inhibited using mitomycin C treatment and the magnetite within MCL-labeled cells was maintained throughout the 7 day culture period. However, the amount of magnetite within proliferating MCL-labeled cells diluted due to cell proliferation. Developing EBs contain a variety of differentiated and undifferentiated cell types with various growth rates. Therefore, we theorized that using MCL-labeled ES cells for EB formation would lead to varied magnetite levels within the cells during EB development and growth. Based on this hypothesis, we report whether a specific cell type can be isolated from developing EBs using magnetic separation of cells labeled with MCLs.

Mouse ES cell line 129sv (Chemicon, Pittsburgh, PA, USA) was cultured on mitotically inactivated MEF feeder cells treated with mitomycin $\mathrm{C}$ for $2 \mathrm{~h}$. Cells were cultured on $0.1 \%$ gelatin 
(Nacalai Tesque, Kyoto, Japan)-coated tissue culture dishes (Greiner Bio-one, Frickenhausen, Germany) in growth medium composed of Knockout-DMEM ${ }^{\mathrm{TM}}$ (Invitrogen, Carlsbad, CA, USA) with 4 mM L-glutamine (Wako Pure Chemical Industries, Osaka, Japan), non-essential amino acids (NEAA; Invitrogen), $100 \mu \mathrm{M}$ 2-mercaptoethanol (Millipore, Billerica, MA, USA), $100 \mathrm{U} / \mathrm{ml}$ penicillin $\mathrm{G}$ potassium (Wako), $50 \mu \mathrm{g} / \mathrm{ml}$ streptomycin sulfate (Wako), 15\%

Knock-out-serum-replacement (Invitrogen) and 1000 U/1 LIF (ESGRO; Millipore). Medium was replaced every day and ES cells were passaged every 2-3 d. MEFs were isolated from the fetuses of 14 d pregnant BALB/c mice and cultured in Dulbecco's modified Eagle's medium (DMEM; Sigma-Aldrich, St. Louis, MO, USA) with 10\% fetal bovine serum (FBS; Biowest, Miami, FL, USA) and $4 \mathrm{mM} \mathrm{L-glutamine.} \mathrm{Cells} \mathrm{were} \mathrm{cultured} \mathrm{at} 37^{\circ} \mathrm{C}$ in a $5 \% \mathrm{CO}_{2}$ incubator. $\mathrm{MCLs}$ were prepared with colloidal magnetite nanoparticles (average particle size $10 \mathrm{~nm}$; Toda Kogyo, Hiroshima, Japan) and a lipid mixture of $N$-( $\alpha$-trimethylammonioacetyl)-didodecyl-D-glutamate chloride, dilauroylphosphatidylcholine and dioleoylphosphatidyl-ethanolamine at a 1:2:2 molar ratio as described previously (5). Magnetite within cells and viable cell numbers were measured with the potassium thiocynate method and trypan blue exclusion, respectively. For magnetic labeling, MCLs were incubated for $2 \mathrm{~h}$ with an ES cell suspension $\left(1 \times 10^{6}\right.$ cells in $1 \mathrm{ml}$ ES cell culture medium $)$ at a net magnetite concentration of $100 \mathrm{pg} / \mathrm{cell}$. For alkaline phosphatase (AP) staining, magnetically-labeled ES cells were cultured on feeder cells for $7 \mathrm{~d}$, then $1 \times 10^{4}$ cells were replated and after a further $3 \mathrm{~d}$ culture, AP staining was performed as described previously (7). Briefly, cells were washed twice with phosphate buffered saline (PBS), fixed in $0.25 \%$ glutaraldehyde for 5 min at room temperature, then exposed to a solution containing naphthol AS-MX phosphate (Sigma) as a substrate and Fast Violet B Salt (Sigma) as a coupler for $20 \mathrm{~min}$ at $37^{\circ} \mathrm{C}$ in a humidified atmosphere containing 5\% $\mathrm{CO}_{2}$. AP-positive cells were counted using microscope images from five fields of view in three separate wells per sample. ES cells with or without magnetic labeling were used to form EBs with the hanging drop method (2). Briefly, ES cell droplets (7000 cells/ml in $15 \mu \mathrm{ES}$ 
medium without LIF) were placed on the lid of a bacterial grade $100 \mathrm{~mm}$ plastic dish (AsOne, Osaka, Japan). The lid was inverted and placed on the bottom half that was filled with PBS, and then incubated at $37^{\circ} \mathrm{C}$ in a $5 \% \mathrm{CO}_{2}$ incubator (designated day 0 ). On day 3, EBs were transferred into 96 well U-bottom plates (Sumitomo Bakelite, Tokyo, Japan) and cultured in DMEM with 15\% FBS, NEAA, $100 \mathrm{U} / \mathrm{ml}$ penicillin $\mathrm{G}$ potassium and $50 \mu \mathrm{g} / \mathrm{ml}$ streptomycin sulfate. For magnetic capture, EBs were dispersed into single cells using trypsin, then resuspended in $1 \mathrm{ml}$ ES cell medium and transferred to $1.5 \mathrm{ml}$ sterile polypropylene tubes. Magnetic force was applied to the tubes using a cylindrical neodymium magnet with a $4000 \mathrm{G}$ surface magnetic induction. The cell capture percentage was determined with the equation: Magnetic cell capture $(\%)=($ the number of viable cells in the pellet) / [(the number of viable cells in the supernatant $)+($ the number of viable cells in the pellet)] $\times 100$. For RT-PCR analysis, total RNA from magnetically captured or non-captured cells was extracted using RNAiso Plus reagent (Takara, Otsu, Japan). RNA was reverse-transcribed into cDNA using $1 \mu \mathrm{g}$ total RNA with a ReverTraAce First Strand cDNA synthesis kit (Toyobo, Osaka, Japan). Specific genes were PCR amplified using primers shown in Table 1. For flow cytometric analysis of mouse vasa homolog (Mvh)-positive germ cells, the cells were fixed in 4\% paraformaldehyde (PFA) for $10 \mathrm{~min}$ and then permeabilized using 90\% methanol (Wako) for $30 \mathrm{~min}$ on ice. Cells were incubated for $1 \mathrm{~h}$ with a primary antibody specific for Mvh (rabbit anti-Mvh antibody; abcam, Cambridge, UK), and then incubated for 30 min with a secondary antibody labeled with Alexa Fluor 488 (Invitrogen). Each step was followed by washing with PBS. Fluorescently labeled cells were analyzed using a flow cytometer (FACSCalibur; BD, Franklin Lakes, NJ, USA). For histological examination, EBs were washed three times with PBS, fixed with 4\% PFA in PBS and embedded in paraffin. Thin sections $(4 \mu \mathrm{m})$ were prepared and blocked with $1 \%$ bovine serum albumin (Wako) in $\mathrm{PBS}$ at $37^{\circ} \mathrm{C}$ for $30 \mathrm{~min}$, followed by incubation overnight at $4^{\circ} \mathrm{C}$ with the anti-Mvh antibody and biotinylated secondary antibody (Dako, Glostrup, Denmark). Thereafter, EBs were incubated at $37^{\circ} \mathrm{C}$ for $30 \mathrm{~min}$ with peroxidase-conjugated streptavidin (Dako). Peroxidase 
activity was visualized by soaking in $0.02 \%$ diaminobenzidine tetrahydrochloride containing $0.005 \%$ hydrogen peroxide at room temperature for $10 \mathrm{~min}$. Iron particles and cellular nuclei within EBs were stained with Berlin blue and hematoxylin, respectively. Each step was followed by three washing steps with Tris-buffered saline (TBS). Stained sections were observed using a microscope (Olympus, Tokyo, Japan). The Mann-Whitney rank sum test was used to evaluate the statistical significance difference, and $p$ values of $<0.05$ were considered statistically significant. Animal experimentation was approved by the Ethics Committee for Animal Experiments of the Faculty of Engineering, Kyushu University (A21-098-1).

As shown in Figure 1A, ES cells rapidly took up MCLs and reached $13 \pm 2.1 \mathrm{pg} / \mathrm{cell}$ at $2 \mathrm{~h}$ after MCL addition. Thereafter, magnetite uptake did not significantly increase and reached a maximum at $4 \mathrm{~h}(18 \pm 2.4 \mathrm{pg} / \mathrm{cell})$. ES cells were cultured in the presence of MCLs for $2 \mathrm{~h}$ in subsequent magnetic labeling experiments. The effects of magnetic labeling on ES cell proliferation and AP-positive colony formation were examined. As shown in Figure 1B, ES cell growth rates with and without MCL labeling were $1.02 \pm 0.02$ and $1.01 \pm 0.02 \mathrm{~d}^{-1}$ (day 2-8), respectively. Thus, magnetic labeling with MCLs at $100 \mathrm{pg} / \mathrm{cell}$ did not affect cell proliferation. Moreover, the AP-positive colony forming assay revealed that magnetic labeling with MCLs did not affect the undifferentiated state of the ES cells (Fig. 1C). On day 8, the average diameter of EBs formed using MCL-labeled ES cells was not significantly different compared with that of EBs without MCL labeling (Fig. 1D). This similarity suggested that ES cell growth and differentiation within EBs was not influenced by MCL labeling $(100 \mathrm{pg} / \mathrm{ml})$ of ES cells. Figure 1E shows the magnetic capture of cells after EB dissociation into single cells. At the beginning of EB formation (day 0), $95 \%$ of cells were magnetically captured suggesting that almost all cells were MCL-labeled and the magnetite uptake of $13 \mathrm{pg} / \mathrm{cell}$ (Fig. 1A) was sufficient for magnetic cell separation. Thereafter, the magnetic cell capture percentage from EBs decreased with EB growth (Fig. 1D) during the $8 \mathrm{~d}$ culture period (Fig. 1E) suggesting that the magnetite within cells was diluted by cell proliferation. The growth rates of cells within EBs were 
not considered uniform because developing EBs contain a variety of differentiated cell types. To investigate whether a specific cell type could be magnetically isolated, EBs on day 3 or 8 were dissociated into single cells and magnetically separated cells were RT-PCR analyzed using primers specific for cell differentiation markers. On day 8 , markers of the three germ lineages were expressed in the magnetically non-captured cells, while only the ectoderm marker (Nestin) was expressed in the magnetically captured cells (Fig. 2A). However, RT-PCR analyses revealed germ cell markers (Mvh, Blimp-1, Fragilis and Stella) were expressed in both magnetically captured and non-captured cells from day 3 (Fig. 2B). It has been reported that the activation of gene expression within EBs during ES cell differentiation occurs in a stepwise manner and germ cell marker genes are expressed in the early stage of embryonic growth (9). Although germ cells have an important role in the transmission of genetic information, the proportion of ES cell differentiation into germ cells is low (8). In the present study, we used Mvh that is specifically expressed in differentiating germ cells including primordial germ cells (PGCs) to evaluate the magnetic separation of germ cells. Toyooka et al. reported in vitro differentiation of mouse ES cells into PGCs and subsequent germ cells using Mvh as the marker (9). We investigated whether Mvh-positive germ cells can be enriched using magnetic separation with MCL-labeled ES cells from developing EBs on day 8. Flow cytometric analysis revealed that Mvh was expressed in $11 \pm 2 \%$ of cells within EBs and magnetic labeling did not affect the percentage of Mvh-positive cells (Fig. 2C). Using magnetic separation, Mvh-positive cells were enriched in the fraction of magnetically captured cells (Fig. 2D). In mouse EBs, how ES cells aggregate and arrange themselves is unknown. To identify the localization of Mvh-positive cells within EBs, cross-sections of EBs on day 8 were stained with an anti-Mvh antibody and Berlin blue (Fig. 2E). As observed, both Berlin blue-positive magnetite particles and Mvh-positive cells were mainly distributed at the center of EBs, and some Mvh-positive cells had internalized the magnetite particles (Fig. 2E). Martin et al. reported that the outer cells of mouse EBs at an early stage correspond to endoderm, while the inner cells correspond to the ectoderm (10). In the present study, 
magnetite particles were spatially distributed in the inner cells of EBs on day 8, and the magnetically captured cells expressed both the ectoderm marker (Nestin, Fig. 2A) and germ cell markers (Fig. 2B). Our analysis suggests that the proliferation of germ cells was slower than the cells of the three germ lineages and the amount of magnetite particles in the outer cells decreased due to progressive cell growth during EB development.

In conclusion, we enriched Mvh-positive germ cells from EBs after $8 \mathrm{~d}$ of differentiation using magnetic separation together with MCL-labeled ES cells. These results indicate that the separation of cells from developing EBs at a specific stage based on their magnetite loading is an alternative method for magnetic cell separation, and this methodology provides a viable tool for studies of ES cell growth and differentiation within EBs.

\section{References}

1. Hübner, K., Fuhrmann, G., Christenson, L. K., Kehler, J., Reinbold, R., De La Fuente, R., Wood, J., Strauss J. F. 3rd, Boiani, M., and Schöler, H. R.: Derivation of oocytes from mouse embryonic stem cells, Science, 300, 1251-1256 (2003).

2. Kurosawa, H.: Methods for inducing embryoid body formation: in vitro differentiation system of embryonic stem cells, J. Biosci. Bioeng., 103, 389-398 (2007).

3. Neurauter, A.A., Bonyhadi, M., Lien, E., Nøkleby, L., Ruud, E., Camacho, S., and Aarvak, T.: Cell isolation and expansion using Dynabeads, Adv. Biochem. Eng. Biotechnol., 106, 41-73, 2007.

4. Ito, A., Shinkai, M., Honda, H., and Kobayashi, T.: Medical application of functionalized magnetic nanoparticles, J. Biosci. Bioeng., 100, 1-11, 2005.

5. Shinkai, M., Yanase, M., Honda, H., Wakabayashi, T., Yoshida, J., and Kobayashi, T.: Intracellular hyperthermia for cancer using magnetite cationic liposomes: in vitro study, Jpn. J. 
Cancer. Res., 87, 1179-1183, 1996.

6. Ito, A., Jitsunobu, H., Kawabe, Y., Ijima, H., and Kamihira, M.: Magnetic separation of cells in coculture systems using magnetite cationic liposomes. Tissue. Eng. Part C. Methods., 15, 413-423, 2009.

7. Horie, M., Ito, A., Kiyohara, T., Kawabe, Y., and Kamihira, M.: E-cadherin gene-engineered feeder systems for supporting undifferentiated growth of mouse embryonic stem cells, J. Biosci. Bioeng, 110, 582-587 (2010).

8. Park, T. S., Galic, Z., Conway, A. E., Lindgren, A., van Handel, B. J., Magnusson, M., Richter, L., Teitell, M. A., Mikkola, H. K., Lowry, W. E., Plath, K., and Clark, A. T.:

Derivation of primordial germ cells from human embryonic and induced pluripotent stem cells is significantly improved by coculture with human fetal gonadal cells, Stem Cells, 27, 783-795 (2009).

9. Toyooka, Y., Tsunekawa, N., Akasu, R., and Noce, T.: Embryonic stem cells can form germ cells in vitro, Proc. Natl. Acad. Sci. U.S.A., 100, 11457-11462 (2003).

10. Martin, G. R.: Isolation of a pluripotent cell line from early mouse embryos cultured in medium conditioned by teratocarcinoma stem cell, Proc. Natl. Acad. Sci. U.S.A., 78, 7634-7638 (1981). 


\section{Figure legends}

FIG. 1. Effect of magnetic labeling with MCLs on ES cells. (A) The uptake of magnetite particles by ES cells after MCL addition (100 pg/cell). (B) Time course of ES cell growth. Open circle, without magnetic labeling; closed circle, with magnetic labeling. (C) AP-positive ES cell colonies. Open column, without magnetic labeling; closed column, with magnetic labeling. (D) Time course of EB growth. Open circle, without magnetic labeling; closed circle, with magnetic labeling. (E) The percentage of magnetically captured cells from dissociated EBs. All data points represent the mean $\pm \mathrm{SD}$ of triplicate experiments.

FIG. 2. Magnetic separation of cells in EBs. (A) RT-PCR analyses of the non-captured or magnetically captured cells for markers of the three germ lineages. (B) RT-PCR analyses of the non-captured or magnetically captured cells for germ cell markers. (C) Flow cytometric analysis of Mvh-positive cells in EBs on day 8 with (closed column) or without (open column) magnetically labeled ES cells. The data points represent the mean $\pm \mathrm{SD}$ of triplicate experiments. (D) Flow cytometric analysis of Mvh-positive cells for the non-captured (open column) or magnetically captured (closed column) cells in EBs on day 8. The data points represent the mean \pm SD of triplicate experiments $* p<0.05$. (E) Cross-sectional observation of an EB with MCL-labeled ES cells on day 8. Mvh was immunohistochemically stained using specific antibodies (in dotted line region). Iron particles and cell nuclei were stained with Berlin blue (arrowheads) and hematoxylin (arrows), respectively. Bar: $25 \mu \mathrm{m}$. 
A

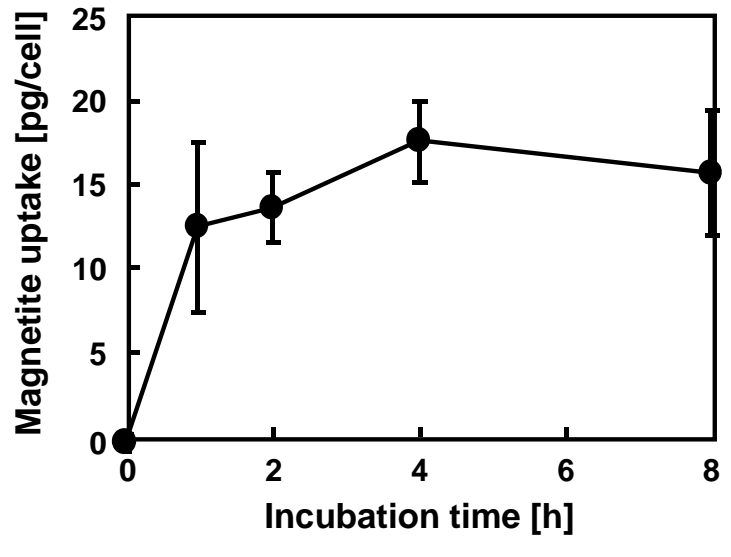

C

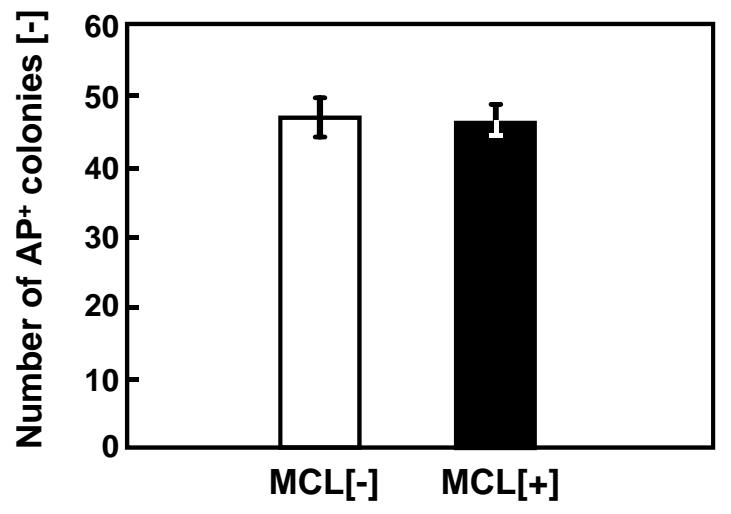

B

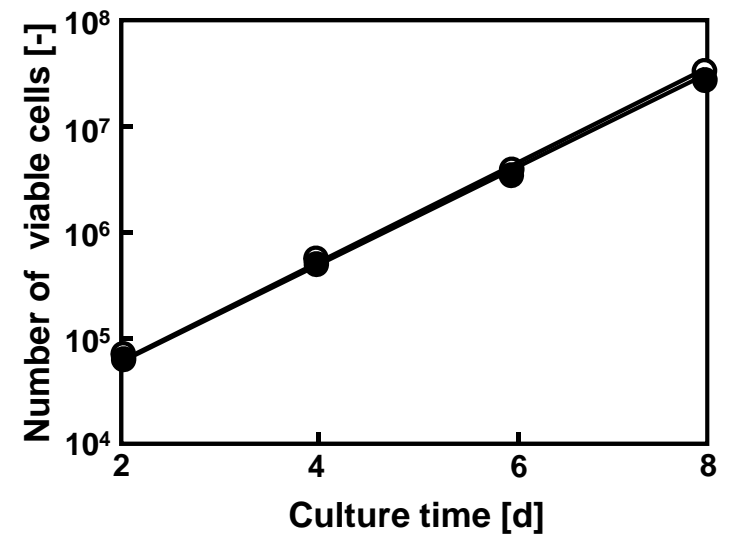

D

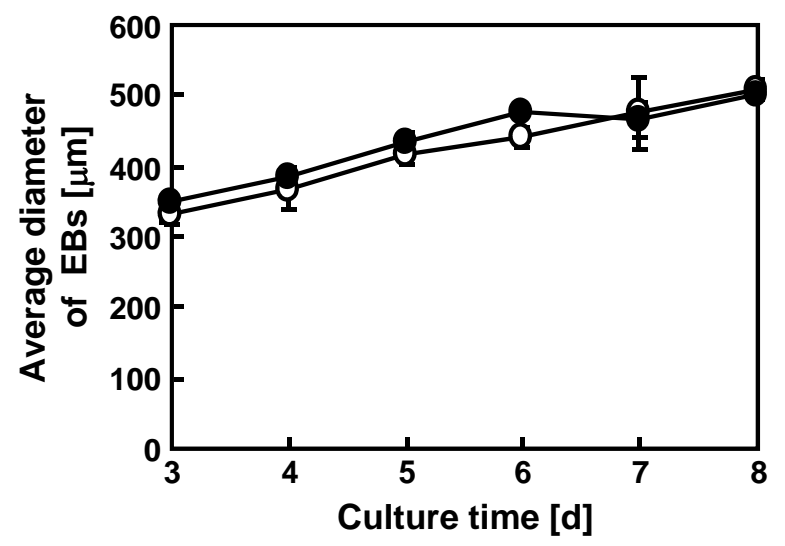

E

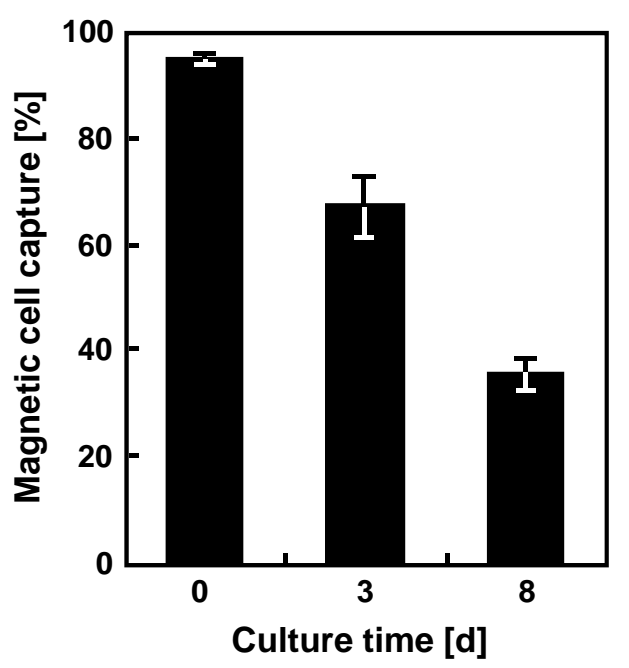

Fig. 1 Horie et al. 


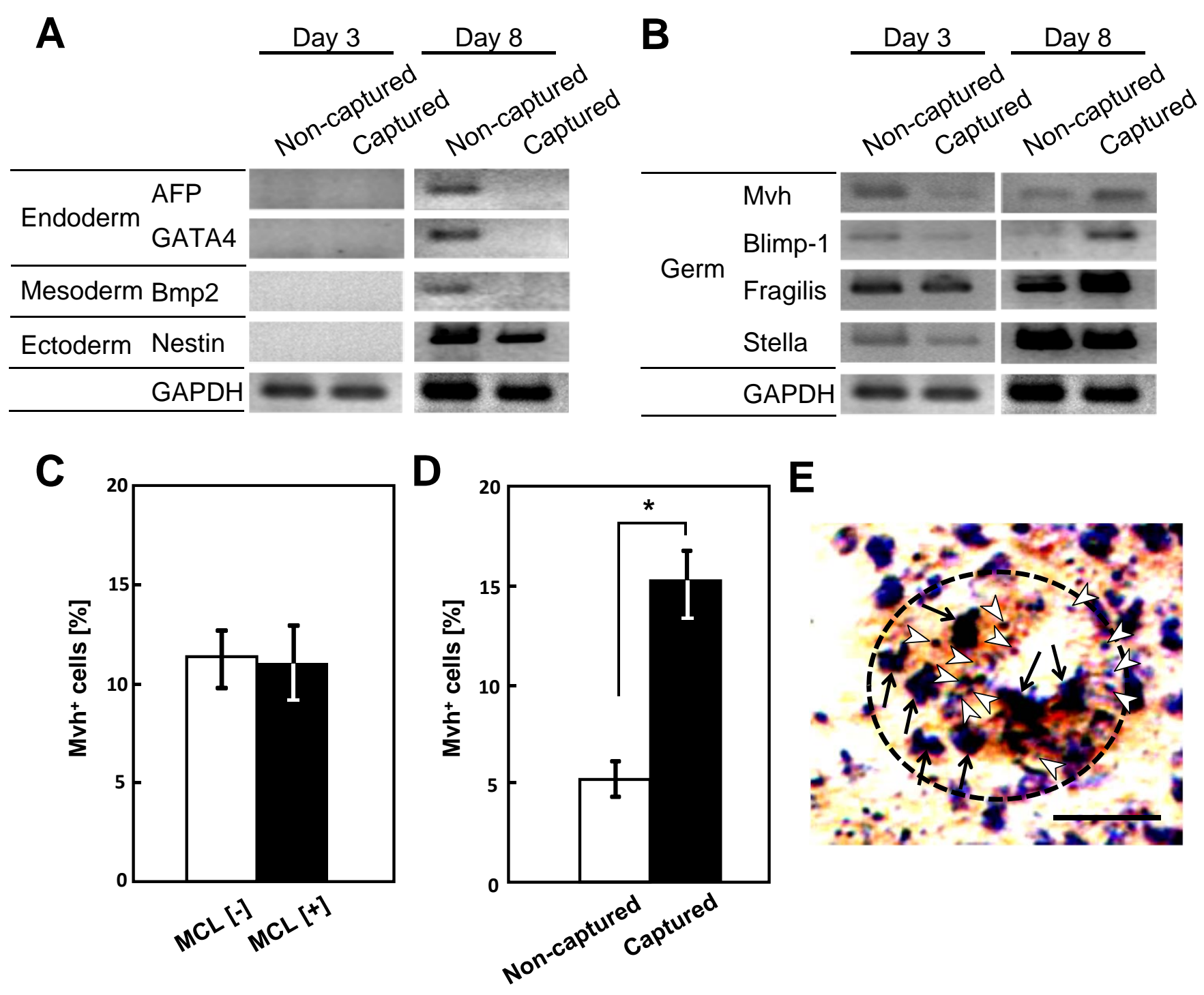

Fig. 2 Horie et al. 